\title{
Phenotypic variability in familial prion diseases due to the D178N mutation
}

\section{J J Zarranz, A Digon, B Atarés, A B Rodríguez-Martínez, A Arce, N Carrera, I Fernández- Manchola, M Fernández-Martínez, C Fernández-Maiztegui, I Forcadas, L Galdos, J C Gómez- Esteban, A lbáñez, E Lezcano, A López de Munain, J F Martí-Massó, M M Mendibe, M Urtasun, J M Uterga, N Saracibar, F Velasco, M M de Pancorbo}

J Neurol Neurosurg Psychiatry 2005;76:1491-1496. doi: 10.1136/jnnp.2004.056606

Background: Between January 1993 and December 2003, 19 patients with familial prion diseases due to the D178N mutation were referred to the regional epidemiological registry for spongiform encephalopathies in the Basque Country in Spain, a small community of some 2100000 inhabitants.

See end of article for authors' affiliations

Correspondence to: Professor J J Zarranz, Neurology Service, Hospital Cruces, Department of Neurosciences, University of the Basque Country, 48903, Baracaldo, Vizcaya, Spain; iizarranz@hcru. osakidetza.net

Received 20 October 2004 Revised version received 9 March 2005 Accepted 25 March 2005 Methods: Ten further patients belonging to the same pedigrees were retrospectively ascertained through neurological or neuropathological records. In four of the patients, the diagnosis was confirmed by analysing DNA obtained from paraffin blocks. In this article, we report on the clinical, genetic, and pathological features of the 23 patients carrying the D178N mutation confirmed by genetic molecular analysis. Haplotyping studies suggest a founder effect among Basque born families, explaining in part this unusually high incidence of the D178N mutation in a small community. Only two patients (8\%) lack familial antecedents.

Results: We have observed a phenotypic variability even among homozygous 129MM patients. Our findings challenge the currently accepted belief that MM homozygosity in codon 129 is always related to a fatal familial insomnia (FFI) phenotype. Indeed, seven out of 17 patients with a 129MM genotype in this series presented with a Creutzfeldt-Jakob disease (CID) clinicopathological picture.

Conclusions: The considerable clinical and pathological overlapping observed among homozygous $129 \mathrm{MM}$ patients favours the view that FFI and CJD178 are the extremes of a spectrum rather than two discrete and separate entities. Other genetic or environmental factors apart from the polymorphism in codon 129 may play a role in determining the phenotypic expression of the DI78N mutation in the PRNP gene.
$\mathrm{T}$ he Basque Country is a small region in the north of Spain of some 2100000 inhabitants. In the period between January 1993 and December 2003, a surprisingly high number of familial cases of prion diseases were referred by neurologists to the registry for spongiform encephalopathies set up by the Basque Government.

The general epidemiological features of prion diseases in the Basque Country have been reported elsewhere. ${ }^{1}$ There are two main possible explanations for the unusually high incidence of familial prion diseases in our small community. First, a comprehensive ascertainment by the neurologists working in the public health system, and second, a probable founder effect of the D178N mutation in the families of Basque origin. ${ }^{2}$

In this article we will focus on the clinical features of the 23 patients with the genetically confirmed D178N mutation emphasising the limited correlation between genotype and phenotype, and the phenotypic variability.

\section{METHODS}

We refer the reader to our previous paper ${ }^{1}$ (for details about ascertainment of patients) and the epidemiological registry. We have adhered to the WHO criteria and recommended guidelines for the diagnosis of prion diseases. ${ }^{3}$ All studies have been approved by the Ethical Committee of the School of Medicine.

Thirteen pedigrees carrying the D178N mutation have been identified. Among them, 19 patients were diagnosed prospectively and 10 further probable cases were ascertained retrospectively. In four of the retrospective cases, the diagnosis was confirmed by analysing DNA obtained from archival paraffin blocks.

Thirteen autopsies have been performed. Immunohistochemistry PrP-res has been carried out in 10 cases and immunoblotting in just three cases (test performed since 2001). Polysomnographic (PSG) recordings have been obtained in 11 patients in a sleep laboratory according to standard procedures.

Patients were classified as having a Creutzfeldt-Jakob disease (CJD) phenotype when dementia, ataxia, myoclonus, and other abnormal movements dominated the clinical picture, and a fatal familial insomnia (FFI) phenotype when insomnia with or without a diurnal dreaming state, hallucinations, delirium, and disautonomia preceded motor symptoms and cognitive deterioration.

\section{RESULTS}

The 19 prospectively confirmed cases linked to the D178N mutation surpass in number the 14 confirmed sporadic cases observed during the same period. Nine families are of Basque origin and the remaining four came from other Spanish regions. Haplotyping studies suggest that at least six of the nine Basque born families are genetically related. ${ }^{2}$

We have analysed the clinicopathological findings of the 19 prospective and the four retrospective genetically confirmed cases. There were 13 women and 10 men. A summary of the main clinical features is presented in table 1 . Mean age at

Abbreviations: CJD, Creutzfeldt-Jakob disease; FFI, fatal familial insomnia; PSG, polysomnography 
它

$\mid$

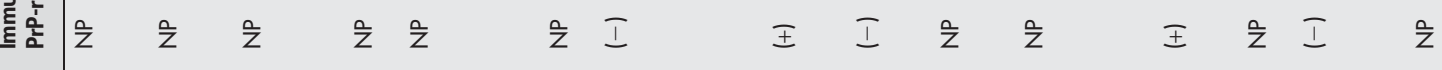

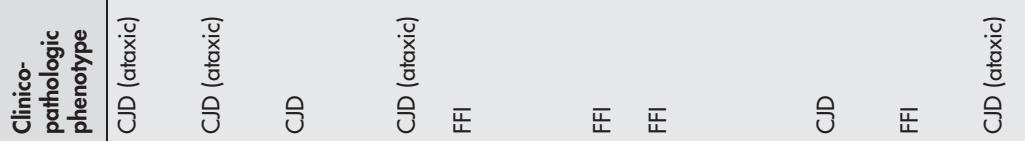

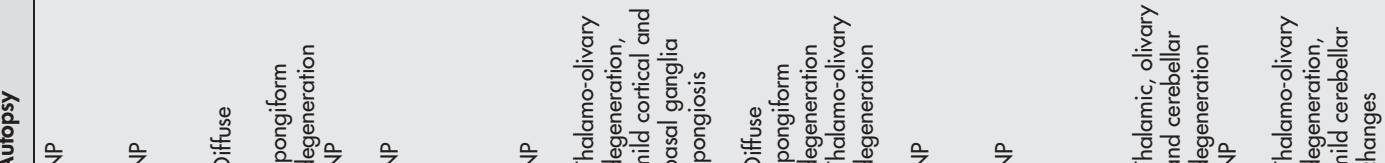

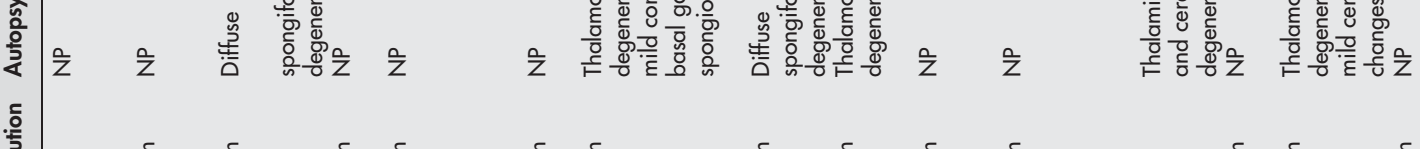

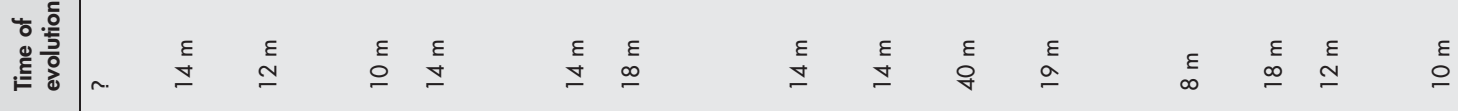

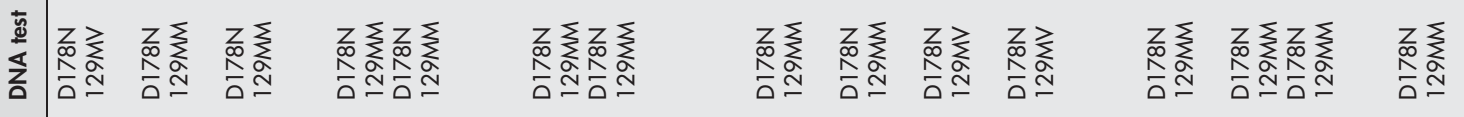

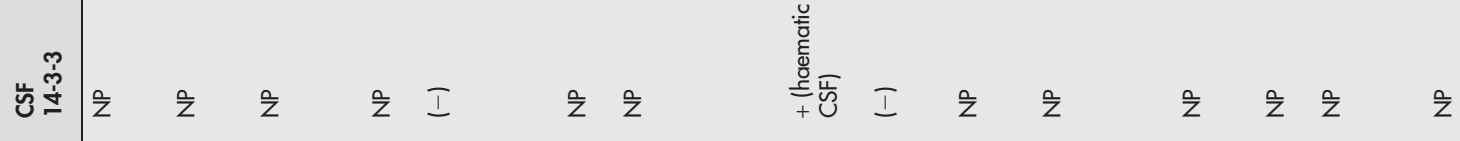

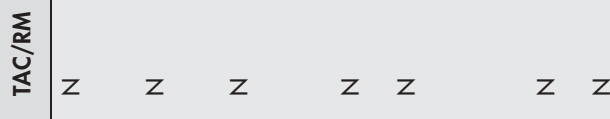

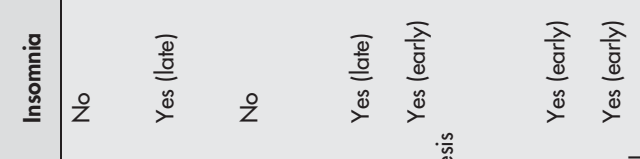

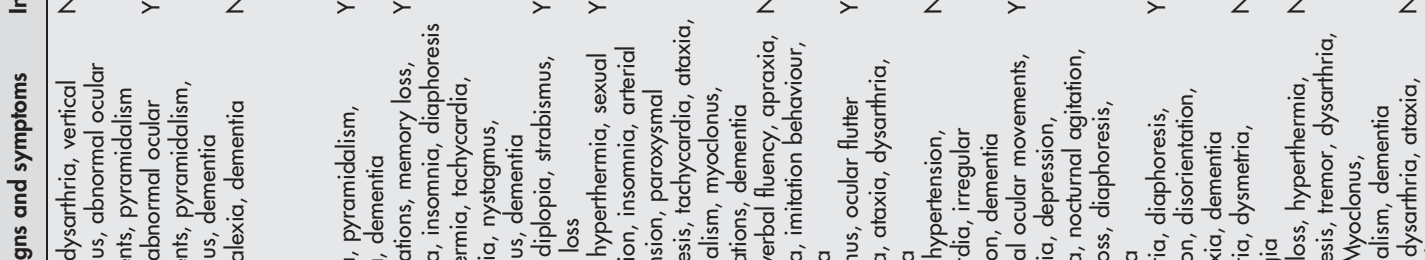

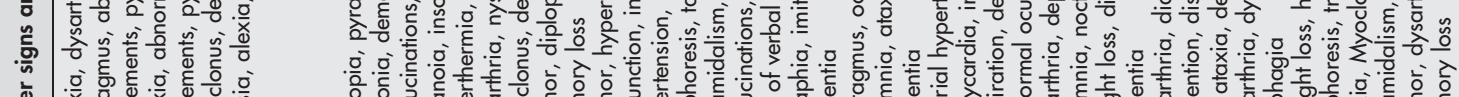

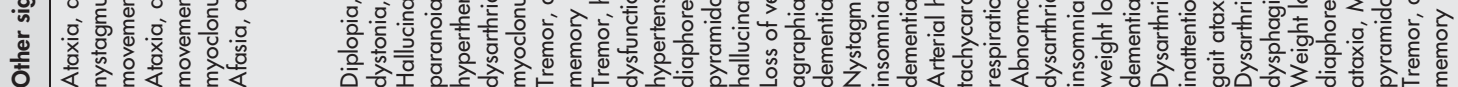

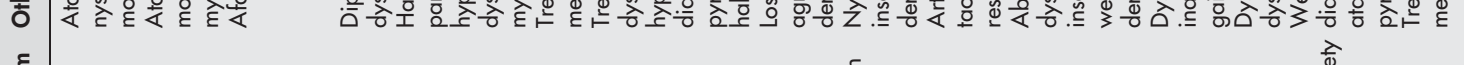

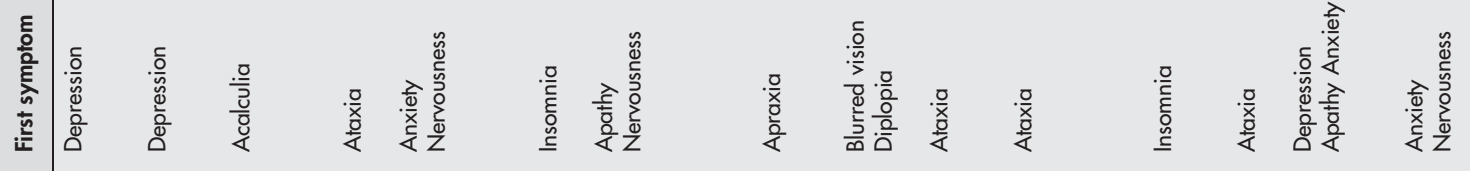
t。

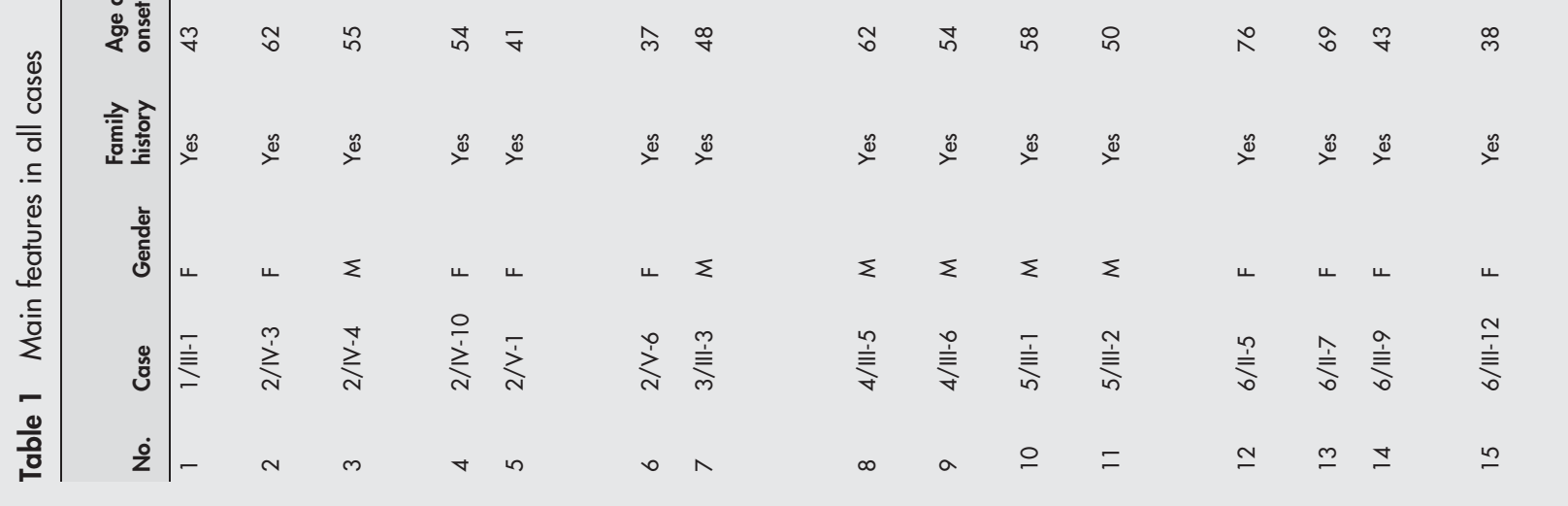




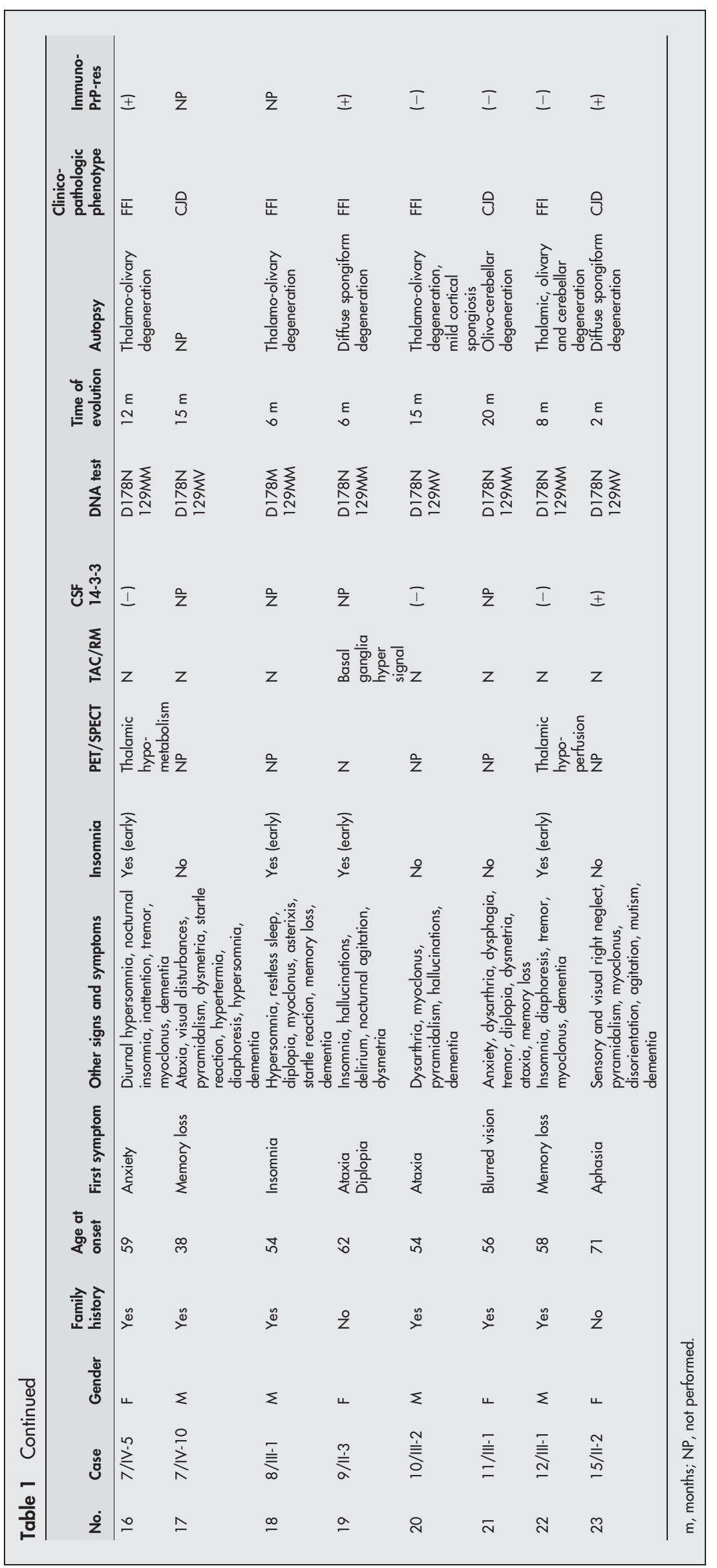


Table 2 First symptom identified by the patients or their relatives

\begin{tabular}{ll}
\hline Anxiety, nervousness, depression, or apathy & 7 \\
Gait ataxia & 6 \\
Insomnia or disturbed sleep & 3 \\
Blurred or double vision & 2 \\
Memory disturbances & 2 \\
Acalculia & 1 \\
Apraxia & 1 \\
Aphasia & 1 \\
\hline
\end{tabular}

onset of the disease was 53.3 years (range: 37-76). The presenting symptoms are summarised in table 2. Sleep loss was the first complaint in only three cases. In addition, insomnia was an early feature in the evolution in another ten patients and a late finding in three more patients. The PSG studies are summarised in table 3.

\section{DISCUSSION}

Three main clinical and pathological phenotypes have been differentiated in familial prion diseases: hereditary CJD, Gertsmann-Sträussler-Sheinker disease, and FFI, ${ }^{4}$ although genotypic and phenotypic heterogeneity is well established. ${ }^{5}$ FFI was described as being associated with the DI78N mutation when the mutant allele encodes methionine in the polymorphism of codon 129, whereas the encoding of valine by the mutant allele would produce the CJD phenotype. ${ }^{6}$ Around 30 FFI families have been reported world-wide so far. $^{7-9}$

Although insomnia is the most characteristic early symptom of the D178N-129M mutation, only three of our patients complained of insomnia as the first symptom. Seven patients presented with gait ataxia. Ill defined psychological changes or cognitive decline were the first symptoms in another seven patients, a finding already pointed out in the literature. ${ }^{10}$ However, it may happen that in the absence of a spontaneous subjective complaint, abnormal nocturnal sleep passes unnoticed. This occurred in four of our cases in which abnormal sleep was detected due to a PSG study because neither the patients nor their relatives reported any trouble with nocturnal sleep. In our series, we were able to carry out a PSG in 11 patients. In four of them, the PSG was obtained relatively early on (less than 6 months from onset of the disease) and showed severe disorganisation of the normal sleep pattern with total absence of physiological sleep rhythms in two of the patients. These findings prove that severe sleep involvement could happen earlier than is signalled in the literature. ${ }^{11}$
The first symptoms exhibited by three patients suggested focal neocortical involvement (acalculia, apraxia, aphasia). It is worth mentioning that the course of the disease in these three patients was rapid and the disease fulfilled the clinical and neuropathological criteria for a diagnosis of CJD, although two of the patients were homozygous for 129MM.

Structural neuroimaging tests (routine brain CT or MRI) were normal in our patients, with the exception of case 9/II-3 in whom the T2 weighted MRI showed a mild basal ganglia hypersignal. However, DWI sequences, which are much more sensitive in detecting cortical and basal ganglia hypersignals, were not systematically obtained. Pseudoperiodic sharp wave activities were not observed in serial EEGs with a single exception in case 15/II-2 whose rapid clinical course and diffuse spongiform degeneration found at autopsy correspond to a classic CJD phenotype.

Detection of the 14-3-3 protein in the CSF has a sensitivity and specificity of over $90 \%$ for the diagnosis of prion disease, especially in the sporadic varieties of CJD. All our cases have been studied in the same laboratory, whose overall results have been published..$^{12}$ In the familial cases the positivity is around $50 \%$, but samples from the carriers of the D178N mutation have always been negative. In our series, only two out of seven 14-3-3 tests have yielded a positive result (cases 4/III-5 and 15/II-2). In case 4/III-5, a false positive result can not be excluded as the spinal fluid was slightly contaminated with blood during the lumbar puncture. Nevertheless, it is worth emphasising that in these two cases the clinical picture and the neuropathological changes were compatible with a diagnosis of CJD.

Homozygosity in codon 129 increases susceptibility to all forms of prion diseases whether sporadic, genetic, or transmissible. This polymorphism plays an important role in modulating the clinico-pathological phenotype. ${ }^{6}$ However, it is evident from our experience and that of other authors ${ }^{13-19}$ that there must be additional factors which modify the phenotype. Padovani et al $^{19}$ compared several variables such as age of onset, duration of disease, histopathology findings, and PrP-res deposits between patients homozygous and heterozygous at codon 129. They concluded that in spite of certain significant differences, there was considerable overlapping between both groups as regards age of onset and disease duration. However, in some studies, duration of illness is significantly shorter in homozygous MM patients than in MV or VV patients. Our series is too small to make a valid comparison as we have observed only six 129MV patients compared with 17 129MM cases (table 4). However, it seems that disease duration is actually longer in $129 \mathrm{MV}$ patients than in 129MM patients. The disease duration is shortest in homozygous 129MM patients with a FFI phenotype. This is in agreement with the findings of the Italian authors. ${ }^{19}$

Table 3 Summary of PSG studies

\begin{tabular}{|c|c|c|c|}
\hline Case & $\begin{array}{l}\text { Time of evolution } \\
\text { (months) }\end{array}$ & Length of study & Main findings \\
\hline 2/IV-10 & 9 & 1 night & Absence of sleep rhythms \\
\hline 2/V-1 & 12 & $24 \mathrm{~h}$ & Absence of sleep rhythms \\
\hline $2 / \mathrm{V}-6$ & 6 & $\begin{array}{l}3 \text { nights } \\
\text { plus } 24 \mathrm{~h}\end{array}$ & $\begin{array}{l}\text { Reduced sleep efficacy; sleep fragmentation; REM without muscle atonia; central apnoeas, } \\
\text { tachycardia; profuse periodic and non-periodic leg movements }\end{array}$ \\
\hline $3 / I I I-3$ & 12 & 1 night & $\begin{array}{l}\text { Severe abnormal sleep pattern; some spindles in phase II; no phases III or IV; a single period of } \\
\text { REM sleep }\end{array}$ \\
\hline $4 / I I I-6$ & 9 & 1 night & Absence of sleep rhythms (phase I only); central apnoeas; periodic legs movements \\
\hline & 12 & 1 night & Absence of sleep rhythms \\
\hline $5 / 111-1$ & 9 & 1 night & Severe abnormal pattern of sleep (phases I and II); very few spindles; central apnoeas \\
\hline $5 / 111-2$ & 3 & 1 night & Severe abnormal pattern of sleep (phases I and II); very few spindles; a single period of REM sleep \\
\hline $6 / 111-9$ & 11 & 1 night & Severe abnormal pattern of sleep; central apnoeas; some fragments of REM sleep \\
\hline $7 / 111-4$ & 12 & 1 night & Absence of sleep rhythms \\
\hline $8 / 111-1$ & 6 & 1 night & Absence of sleep rhythms \\
\hline $9 / 11-2$ & 6 & 1 night & Absence of sleep rhythms \\
\hline
\end{tabular}


Table 4 Influence of the polymorphism in codon 129 over the phenotype in carriers of the D178N mutation

\begin{tabular}{llllll}
\hline $\begin{array}{l}\text { Genotype polymorphism, } \\
\text { codon 129 }\end{array}$ & $\begin{array}{l}\text { Number of } \\
\text { patients }\end{array}$ & Phenotype & $\begin{array}{l}\text { Number of } \\
\text { patients }\end{array}$ & $\begin{array}{l}\text { Mean age at onset, } \\
\text { years (range) }\end{array}$ & $\begin{array}{l}\text { Mean duration of illness, } \\
\text { months (range) }\end{array}$ \\
\hline WV & 0 & - & - & - & - \\
MV & 6 & CJD & 4 & $52.5(38-71)$ & $19(2-40)$ \\
& & 2 & $52(50-54)$ & $17(15-19)$ \\
MMI & CJD & 7 & $56.5(38-69)$ & $14.8(10-20)$ \\
& 17 & FFI & 10 & $63.2(37-76)$ & $10.8(6-18)$ \\
\hline
\end{tabular}

Seven of the homozygous 129MM patients had a CJD phenotype instead of a FFI phenotype. The differences in age at onset or duration of illness did not reach statistical significance.

In our series, although the well known rule that the FFI phenotype is linked to the D178M/129MM genotype ${ }^{6}$ always seems to hold, the reverse is not true, as seven patients with the same genotype had a clinical and neuropathological profile compatible with CJD (table 4). The same conclusion can be reached from the findings in the heterozygous MV patients. In four of the six heterozygous MV patients, the mutant allele was coupled with $\mathrm{M}$ in the 129 position. However, the clinicopathological phenotype was FFI in two cases and CJD in the other two. In the remaining two 129MV patients with a typical CJD phenotype, the position of $\mathrm{M}$ in codon 129 was uncertain.

Other environmental or genetic factors, apart from the polymorphism in codon 129, must therefore influence the phenotypic expression of the D178N mutation. We can not correlate the phenotype or genotype with the PrP-res isotype as only three inmmunoblots have been performed in these patients.

In conclusion, we report on the largest world-wide series of the D178N mutation in the PRNP gene, observed in a small community. We emphasise the absence of a clear cut correlation between genotype and phenotype and the phenotypic variability. The considerable clinical and pathological overlapping observed even among homozygous 129MM patients favours the view that FFI and CJD178 are the extremes of a spectrum rather than two discrete and separate entities.

\section{ACKNOWLEDGEMENTS}

We wish to thank the following people for their valuable cooperation in this study. Dr L González de Galdeano and Dr JM Arteagoitia for allowing us access to the data of the Epidemiological Registry for Prion Disease of the Health Department of the Basque Government. The genetic studies were performed in the laboratories of Professor $\mathrm{J}$ Collinge, Dr N Cuadrado, Dr F Coria, Dr R Yagüe, and Professor Marian Martinez de Pancorbo. The 14-3-3 protein test was carried out in the laboratory of Dr F Graus and Dr A Sainz. Professor I Ferrer helped us with the neuropathological studies. The polysomnographic recordings were carried out and interpreted by Dr P Madoz, Dr J Zamacona, and Dr AI Fernández-Bedoya.

\section{Authors' affiliations}

J J Zarranz, M Fernández-Martínez, C Fernández-Maiztegui, I Forcadas, J C Gómez-Esteban, E Lezcano, M M Mendibe, F Velasco, Neurology Service, Hospital Cruces, Department of Neurosciences, University of the Basque Country, Baracaldo, Vizcaya, Spain A Digon, Neurology Service, Hospital Santiago Apóstol, Vitoria, Alava, Spain

B Atarés, Service of Pathology, Hospital Txagorritxu, Vitoria, Alava, Spain

A B Rodríguez-Martínez, M M de Pancorbo, Department of Zoology and Molecular Biology, School of Pharmacy, University of the Basque Country, Vitoria, Alava, Spain

A Arce, Neurology Service, Hospital Bidasoa, Irun, Guipúzcoa, Spain N Carrera, I Fernández-Manchola, A López de Munain, J F MartíMassó, M Urtasun, Neurology Service, Hospital Donostia, Department of Neurosciences, University of the Basque Country, San Sebastián, Guipúzcoa, Spain

L Galdos, A Ibáñez, Neurology Service, Hospital Txagorritxu, Vitoria, Alava, Spain
J M Uterga, Neurology Service, Hospital Basurto, Bilbao, Vizcaya, Spain

N Saracibar, Service of Pathology, Hospital Santiago Apóstol, Vitoria, Alava, Spain

Competing interests: none declared

\section{APPENDIX}

\section{ADDITIONAL DATA}

Representative cases are reported in more detail below.

\section{Family 2}

\section{Case 2/IV-4}

There was a history of an undefined neurodegenerative condition in the maternal family of this male patient. He first noticed symptoms abruptly at 55 years of age: while on holiday, the patient realised he could not add up the bill in a restaurant. In the following weeks, the acalculia worsened rapidly and was associated with disturbances in speech, writing, and reading. Complementary testing (EEG, CT scan, $\mathrm{CSF}$ analysis) was negative. In the following months, a global cognitive decline occurred and the patient's condition progressively deteriorated. No sleep abnormalities were detected by his relatives. He died after 12 months' illness. On post-mortem examination, diffuse neuronal loss, gliosis, and spongiform degeneration of the cerebral cortex, thalamus, and basal ganglia were confirmed. No selective involvement of the medial nuclei of the thalamus or the inferior olivary nuclei was present. Some PAS positive kurulike plaques were observed in the molecular layer of the cerebellum. Immunohistochemistry for PrP-res was not done. A final clinicopathological diagnosis of familial CJD was made. A molecular genetic analysis of DNA obtained from post-mortem cerebral tissue disclosed a D178N-129MM genotype.

\section{Case 2/V-6}

This woman requested genetic diagnosis on learning of the nature of her family's disease, and it was confirmed that she was a carrier of the D178N-129MM mutation. At 37 years of age she began to complain of broken restless sleep which was confirmed by her husband. Taking benzodiazepines did not improve the quality of her sleep. After 6 months' evolution, while neurological examination and mental status were still normal, three PSG recordings on consecutive nights followed by a continuous $24 \mathrm{~h}$ recording were performed. Severe insomnia was confirmed. All phases of NREM and REM sleep could be identified but in reduced proportion. She had periods of REM sleep without muscle atonia, frequent body movements, and a profusion of periodic and non-periodic leg movements. Her heart rate was continuously raised. Respiration was very irregular with long periods of central apnoea. Nor-adrenaline and adrenaline levels were elevated, while dopamine was in the normal range. In the following weeks she began to notice an action tremor in both hands and intermittent double vision. Her eyes squinted without 
discernible oculomotor paresis. Two months later instability of gait and memory disturbances appeared. A cerebral SPECT-HMPAO detected bilateral thalamic hypo-perfusion. Three months later she died suddenly at home and a postmortem examination was not performed.

\section{Family 4}

\section{Case 4/III-5}

There was a history of dementia in the paternal family of this patient, although his father had died of pulmonary disease at 37 years of age with no neurological symptoms. At 62 years of age, the patient began to experience apraxia when attempting tasks such as writing, dressing, doing up buttons, or driving. Five months later, he was suffering from a complex syndrome with bradypsychia, constructive apraxia, agraphia, low verbal fluency with good comprehension, exaggerated prehension reflexes, imitation behaviour, poor following of Luria's motor sequences, and reduced digit span. The clinical picture suggested a cortical type of degenerative dementia. However, memory and visual and auditory gnosias were normal. No abnormalities of sleep were observed, so PSG was not requested. Cranial CT and MRI were normal. The 14-3-3 protein test was positive, but the CSF was contaminated by blood during the lumbar puncture. Genetic analysis confirmed the presence of the D178N-129MM mutation in the PRNP gene. The patient worsened progressively and died after approximately 18 months of illness. On post-mortem examination, diffuse and severe spongiform degeneration of the cortex and basal ganglia associated with massive neuronal loss and astrocytosis compatible with a neuropathological diagnosis of CJD was found. Immunohistochemistry for PrP-res was strongly positive following a strip-like pattern in the molecular layer of the cerebellum, and was slightly positive in a synaptic pattern in the neocortical areas.

\section{Case 4/III-6}

This patient was a 54 year old man whose disease started almost simultaneously with that of his elder brother, case 4/ III-5. His first symptom was an irregular visual disturbance resulting in doubling of objects. A little while later he was noticed to have insomnia with abnormal movements during the night. To this was added a state of nervousness and insecurity on walking. On examination, there was ocular flutter, ataxic gait, segmental myoclonus, exaggerated startle reaction, dysarthria, and bradypsychia. Basal EEG and MRI were normal. SPECT-HMPAO showed relative hypo-perfusion in the left fronto-temporal areas and both thalami. CSF analysis was normal and the 14-3-3 protein assay was negative. PSG performed 9 months after the beginning of the disease disclosed severely disorganised sleep. Only phase I of NREM sleep was identified, occupying $41 \%$ of the nocturnal recording. PSG was repeated 3 months later and showed a total absence of sleep rhythms. Analysis of DNA showed a D178N-129MM genotype. The patient's condition deteriorated rapidly and he died after 14 months' illness. On postmortem examination, a typical neuropathological pattern of FFI was found. Selective degeneration of the ventral anterior and medio-dorsal thalamic nuclei and inferior olivary nuclei with severe neuronal loss and prominent astrogliosis but without noticeable spongiosis was observed. Immunohistochemistry for PrP-res was positive, in a strip-like pattern, and restricted to the molecular layer of the cerebellum.

\section{Family 9}

Case $9 / 11-3$

This female patient did not cite a family history of neurological disorders; her parents had died without neurological symptoms, the father at 85 and the mother at 87 years of age. At 62 years of age, she began to complain of unsteady gait and double vision and within a few weeks was unable to walk without assistance. Four months after the first symptoms she was admitted to hospital because of severe delirium with visual hallucinations, confusion of reality, insomnia, and nocturnal wandering. Language was normal and during lucid wakeful periods her mental status was near normal (MMSE 32/35). Neurological examination disclosed dissociated nystagmus, vertical gaze paresis, some slight perioral myoclonus, cephalic tremor, severe dysmetria of the four limbs, and gross trunk and gait ataxia. PSG confirmed the absence of any discernible physiological sleep rhythms. Cerebral SPECT-HMPAO was within normal limits. T2 weighted cranial MRI showed a mild to moderate hyper signal of both caudate and putamen. The patient suffered a rapid downhill course to a vegetative state and died 6 months after the beginning of the disease. Neuropathological examination disclosed severe neuronal loss, gliosis, and spongiosis in the thalamus not restricted to the medial nuclei. In addition, there was typical diffuse spongiform degeneration affecting the neocortex, basal ganglia, and molecular layer of the cerebellum compatible with a diagnosis of CJD. Immunohistochemistry for PrP-res was positive following a synaptic pattern with punctated deposits in the deep layers of some neocortical areas, in the thalamus, and in the molecular layer of the cerebellum. DNA analysis obtained from a post-mortem cerebral sample, showed an unsuspected DI78N mutation coupled with the MM homozygosis at codon 129.

\section{REFERENCES}

1 Zarranz JJ, Digon A, Atares B, et al. Familial prion diseases in the Basque Country (Spain). Neuroepidemiology 2005;24(1-2):103-9.

2 Rodriguez Martinez AB, Barreau C, Coupry l, et al. Ancestral origins of the prion protein gene D178N in the Basque Country. Hum Genet 2005, April 2 [Epub ahead of print].

3 WHO. WHO manual for surveillance of human transmissible spongiform encephalopathies including variant Creutzfeldt-Jakob disease. Geneva: Word Health Organization, 2003.

4 Lugaresi E, Medori R, Montagna $\mathrm{P}$, et al. Fatal familial insomnia and dysautonomia with selective degeneration of thalamic nuclei. N Engl J Med 1986;315(16):997-1003.

5 Collinge J, Palmer M. Human prion diseases. In: Palmer M, ed. Prion diseases. Oxford: Oxford University Press, 1997: 18-56.

6 Goldfarb LG, Petersen RB, Tabaton M, et al. Fatal familial insomnia and familial Creutzfeldt-Jakob disease: disease phenotype determined by a DNA polymorphism. Science 1992;258(5083):806-8.

7 Kovacs GG, Trabattoni G, Hainfellner JA, et al. Mutations of the prion protein gene phenotypic spectrum. J Neurol 2002;249(11):1567-82.

8 Montagna P, Gambetti P, Cortelli P, et al. Familial and sporadic fatal insomnia. Lancet Neurol 2003;2(3):167-76.

9 Spacey SD, Pastore M, McGillivray B, et al. Fatal familial insomnia: the first account in a family of Chinese descent. Arch Neurol 2004;61(1):122-5.

10 Almer G, Hainfellner JA, Brucke T, et al. Fatal familial insomnia: a new Austrian family. Brain 1999;122(Pt 1):5-16.

11 Sforza E, Montagna $P$, Tinuper $P$, et al. Sleep-wake cycle abnormalities in fatal familial insomnia. Evidence of the role of the thalamus in sleep regulation. Electroencephalogr Clin Neurophysiol 1995;94(6):398-405.

12 Saiz A, Nos C, Yague J, et al. The impact of the introduction of the 14-3-3 protein assay in the surveillance of sporadic Creutzfeldt-Jakob disease in Catalonia. J Neurol 2001;248(7):592-4.

13 Manetto V, Medori R, Cortelli $P$, et al. Fatal familial insomnia: clinical and pathologic study of five new cases. Neurology 1992;42(2):312-9.

14 Zerr I, Giese A, Windl O, et al. Phenotypic variability in fatal familial insomnia (D178N-129M) genotype. Neurology 1998;51(5):1398-405

15 Reder AT, Mednick AS, Brown P, et al. Clinical and genetic studies of fatal familial insomnia. Neurology 1995;45(6):1068-75.

16 McLean CA, Storey E, Gardner RJ, et al. The D178N (cis-129M) "fatal familial insomnia" mutation associated with diverse clinicopathologic phenotypes in an Australian kindred. Neurology 1997;49(2):552-8.

17 Johnson MD, Vnencak-Jones CL, McLean MJ. Fatal familial insomnia: clinical and pathologic heterogeneity in genetic half brothers. Neurology 1998;51(6): 1715-7.

18 Taniwaki Y, Hara H, Doh-Ura K, et al. Familial Creutzfeldt-Jakob disease with D178N-129M mutation of PRNP presenting as cerebellar ataxia without insomnia. J Neurol Neurosurg Psychiatry 2000;68(3):388.

19 Padovani A, D'Alessandro M, Parchi P, et al. Fatal familial insomnia in a new Italian kindred. Neurology 1998;51(5):1491-4. 